\title{
PRODUCTION AND EVALUATION OF LEUKOCYTE- AND THROMBOCYTE-RICH FIBRIN MEMBRANES IN BIRDS
}

\author{
Laís L. FERnANDES ${ }^{1}$, Sheila C. RAHAL ${ }^{*}$, Alexandre T. FABRO ${ }^{2}$, Sabrina S. BATAH ${ }^{3}$, \\ Alicia G. HIPPÓLITO ${ }^{1}$, Jacqueline M. BISCA ${ }^{1}$, Inajara N. HIROT ${ }^{1}$ and Carlos R. TEIXEIRA ${ }^{1}$ \\ ${ }^{1}$ Department of Veterinary Surgery and Anaesthesiology, School of Veterinary Medicine \\ and Animal Science, São Paulo State University (UNESP), Rubião Júnior s/n, Botucatu \\ 18618-681, São Paulo, Brazil; ${ }^{2}$ Department of Pathology and Legal Medicine, Ribeirão \\ Preto School of Medicine, University of São Paulo (USP), São Paulo, Brazil; ${ }^{3}$ Biological \\ Sciences, Faculty of Philosophy, Sciences and Literature of Ribeirão Preto, USP, Brazil
}

(Received 3 November 2018; accepted 2 May 2019)

The aim of this study was the preparation and histological evaluation of Leukocyte- and Thrombocyte-Rich Fibrin (L-TRF) membranes obtained from the blood of four bird species. Forty adult healthy birds were divided into four groups of equal size: G1 - macaws, G2 - domestic chickens, G3 - parrots, G4 - toco toucans. A total of $0.5 \mathrm{~mL}$ of blood was collected from each bird, put into a glass tube without anticoagulant and centrifuged at $3000 \mathrm{rpm}$ for $10 \mathrm{~min}$. L-TRF membranes produced after compression of the clot were processed for histological analysis. The ratio of thrombocytes/area was not significantly different among Groups G2, G3 and G4, but a significant difference was found between Groups G1 and G2 with the highest thrombocyte concentration/area in G1. The groups did not differ statistically in the number of leukocytes/area. The fibrin-to-cells ratio did not vary statistically among Groups G1, G2 and G3, but this ratio was significantly higher in Group G4 than in the other groups. The thrombocyte-toleukocyte ratio was the highest in Group G1, but it did not differ among Groups G2, G3 and G4. In conclusion, the centrifugation protocol allowed the production of L-TRF membranes in the four bird species studied. Histologically, cell ratios were analogous in domestic chickens and parrots, and macaws had the highest ratio of thrombocytes.

Key words: Avian, protocol, histology, fibrin membrane, L-TRF

Platelet-rich fibrin (PRF) is an autologous fibrin matrix that supports leukocytes and platelets (Borie et al., 2015; Miron et al., 2017). PRF has advantages over platelet-rich plasma (PRP) as it is easier, faster and more affordable to prepare (Ehrenfest et al., 2009; Giannini et al., 2015; Miron et al., 2017). In addition, PRF does not require chemical manipulation of the blood with anticoagulants, or the use of bovine thrombin or calcium chloride, which confirm its use-

*Corresponding author; E-mail: sheila.canevese-rahal@unesp.br;

Phone: 0055 (14) 3880-2041 
fulness in daily clinical practice (Ehrenfest et al., 2009; Giannini et al., 2015; Miron et al., 2017).

PRF is basically composed of fibrin, platelets, growth factors, and different cell types such as stem cells and leukocytes (Hartshorne and Gluckman, 2016a). When applied to the tissue, PRF dissolves slowly while growth factors and cytokines are released gradually over a period of 10 days (Miron et al., 2017). The fibrin produced by PRF has spatial conformation that serves as a substrate for platelets as well as a chemotactic signal for stem cells (Giannini et al., 2015).

The production of PRF is also based on whole blood centrifugation, but the ultimate goal is the production of a fibrin-based biomaterial and not a liquid platelet suspension (Bielecki and Ehrenfest, 2012). The characteristics of the centrifuge and adopted protocols have significant influence on the architecture of fibrin, cells, and growth factors (Hartshorne and Gluckman, 2016b).

PRF can be used in a gel or membrane form to promote wound healing, bone growth, graft stabilisation and haemostasis, among others (Saluja et al., 2011). Due to the three-dimensional architecture of the fibrin matrix, the membrane has tense-elastic consistency, flexibility and great density, which facilitate suturing and handling (Giannini et al., 2015; Hartshorne and Gluckman, 2016c). PRF has been used in human patients for oral, maxillofacial, plastic and otologic surgeries, among other applications (Ehrenfest et al., 2009). The products can be classified into pure platelet-rich fibrin (P-PRF) or leucocyte- and platelet-rich fibrin (L-PRF), according to the absence or presence of leukocytes in the preparations (Dhurat and Sukesh, 2014; Mautner et al., 2015; Lansdown and Fortier, 2017).

Birds may benefit from these types of preparations when they suffer injuries as fractures and tissue loss due to ballistic projectiles, trauma, predation, and domestic accidents, among others (Forbes and Guzman, 2017). However, given the scarcity of relevant avian studies (Mudroňová et al., 2014), the quality of blood concentrates must be well controlled to ensure their safe use.

In this context, this study was aimed at the preparation and histological evaluation of Leukocyte- and Thrombocyte-Rich Fibrin (L-TRF) membranes obtained from the blood of four bird species. The hypothesis was that the membranes could vary histologically among the species even when the same protocol is used.

\section{Materials and methods}

This study was approved by the Ethics Committee for the Use of Animals at the School of Veterinary Medicine and Animal Science (CEUA - no. 79/2016) at São Paulo State University (UNESP), Campus of Botucatu - Brazil, as well as by the National Environmental Wildlife Bureau (SISBIO - no. 53876-1) in Brasília - Distrito Federal, Brazil. 
Forty adult healthy birds were divided into four groups of equal size: G1 10 macaws (Ara ararauna), G2 - 10 domestic chickens (Gallus gallus domesticus), G3 - 10 parrots (Amazona aestiva), and G4 - 10 toco toucans (Ramphastos toco). These birds had been routinely assisted by veterinarians, and immediately before the study, they underwent physical examination and haematological analysis to confirm their health status.

\section{Procedure for Leukocyte- and Thrombocyte-Rich Fibrin (L-TRF) membrane preparation}

A total of $0.5 \mathrm{~mL}$ of blood was collected from the right jugular vein of each bird under physical restraint. A 24-gauge needle (BD Vacutainer ${ }^{\mathbb{B}}$ ) attached to a 3 -mL syringe was used to collect the blood. The birds were not subjected to fasting.

Each blood sample was immediately transferred into a sterile $1.5-\mathrm{mL}$ glass tube without anticoagulant and centrifuged at $3000 \mathrm{rpm}$ for $10 \mathrm{~min}$ using a tabletop centrifuge (Excelsa Baby, model 206, Fanem ${ }^{\circledR}$ ). The L-TRF clot was gently removed from the tube with a pair of tweezers, and a red blood cell (RBC) layer was partially removed with a no. 11 surgical scalpel blade (Fig. 1a). The coat was transformed into an L-TRF membrane by compressing between two surgical compresses moistened with sterile saline solution (Fig. 1b). The membranes were fixed in $10 \%$ neutral buffered formalin for $48 \mathrm{~h}$, and then transferred to a solution containing alcohol $(70 \%)$ for $48 \mathrm{~h}$. After that, the membranes were diaphonised in xylol, and embedded in paraffin for the preparation of serial $4-\mu \mathrm{m}$ thick sections. The sections were stained with haematoxylin and eosin.

Morphometric analyses were carried out based on histomorphometric analyses of an area fraction, which was customised in our previous study (Sant'Ana et al., 2018). Briefly, ten random images were taken of each haematoxylin and eosin stained section (Fig. 1c) at high-power-field (400-fold magnification) using an optical microscope (Olympus BX51) equipped with a camera (CCD Sony) and image analysis software (ProPlus). The total area measured by the analyser in each field was $68,265,680 \mathrm{~mm}^{2}$. The area corresponding to different cell morphologies was measured through manual selection with subsequent measurement of the digitised area. Then, the membranes were analysed for the number of cells per unit area to obtain more accurate identification of their composition. Thrombocytes/area, leukocytes/area, ratio of fibrin to cells, and ratio of thrombocytes to leukocytes were specifically evaluated.

\section{Statistical analysis}

The following descriptive statistical parameters were calculated for each group: mean, standard deviation, median, minimum, maximum and coefficient of variation of all continuous variables. Box-plot graphs were constructed to represent continuous variables and to observe data distribution, verifying symmetry or asymmetry. 

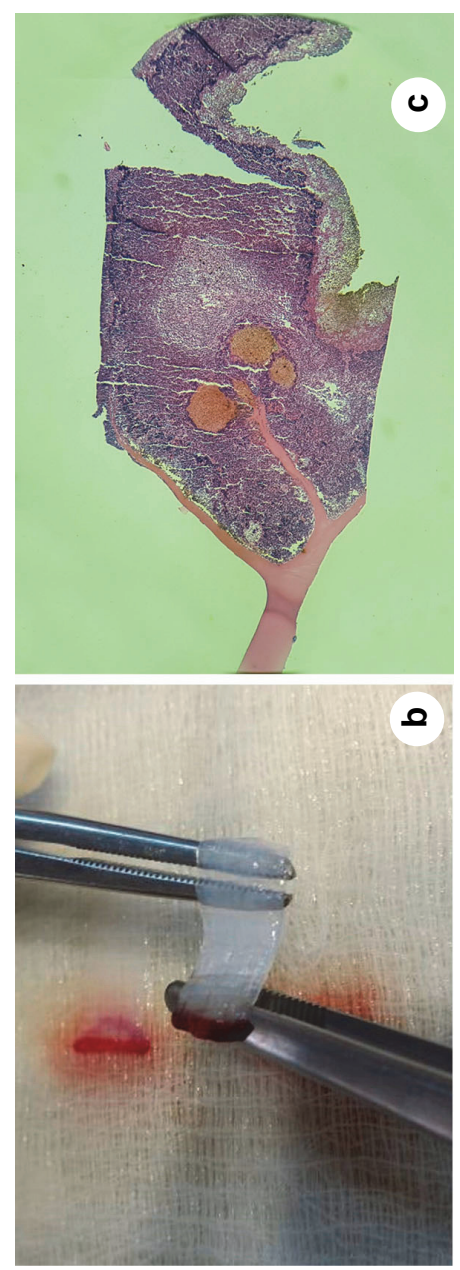

-

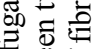

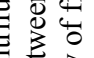

일

迡.

気 离

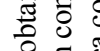

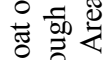

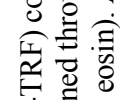

当 뮹

클

局 대유.

휼 है है

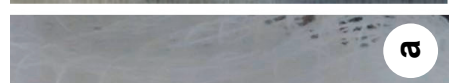

(

空

实

븝 붕

空

定:

ङ

西

\%

要

釜画

in

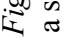


As for the univariate statistical analysis, the continuous variables were analysed using Analysis of Variance (ANOVA) to detect differences in the means of the groups. When a significant difference was verified among the groups, a multiple comparisons test (Tukey's test) was carried out. Differences were considered statistically significant when $\mathrm{P}<0.05$.

\section{Results}

The L-TRF membranes were flexible and apparently resistant in all groups. The thrombocytes/area ratio was not significantly different among Groups G2, G3 and G4; however, thrombocyte concentration/area was statistically higher in G1 than in G2 (Fig. 2). No statistically significant differences were found among the groups regarding the number of leukocytes per unit area (Fig. 3). The fibrin-tocells ratio did not differ significantly among G1, G2 and G3, but G4 differed significantly from the other groups (Fig. 4). The thrombocytes to leukocytes ratio differed significantly between G1 and G2, but not among Groups G2, G3 and G4 (Fig. 5).

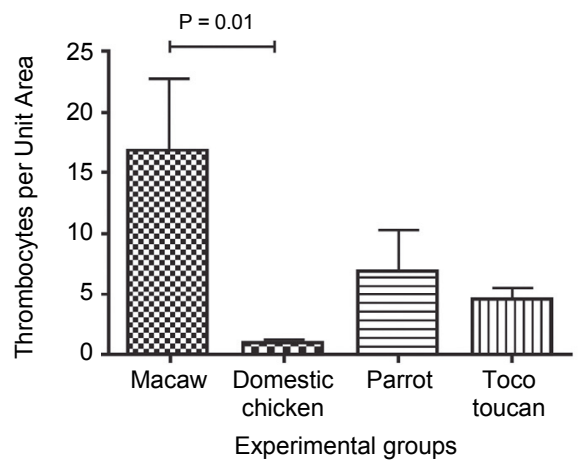

Fig. 2. Number of thrombocytes per unit area observed in L-TRF membranes according to group $(\mathrm{n}=10)$

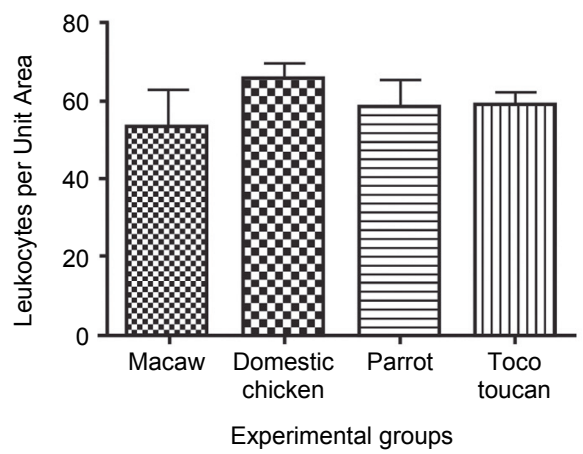

Fig. 3. Number of leukocytes per unit area observed in L-TRF membranes according to group $(\mathrm{n}=10)$ 


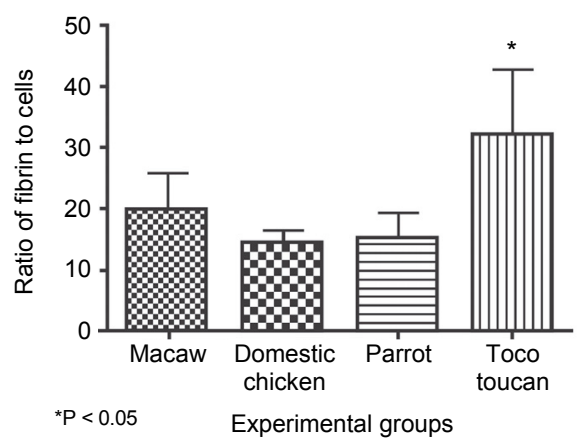

Fig. 4. Ratio of fibrin to cells observed in L-TRF membranes according to group $(\mathrm{n}=10)$

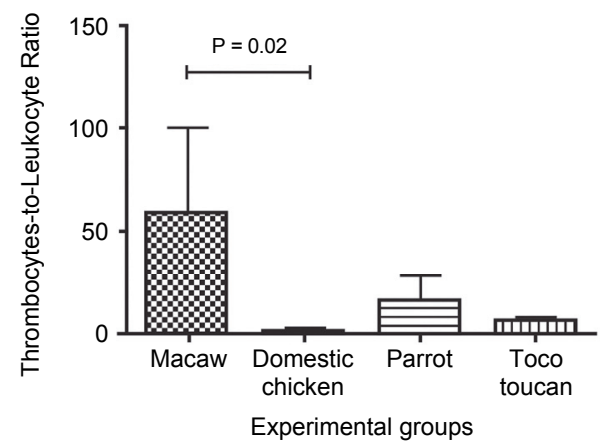

Fig. 5. Ratio of thrombocytes to leukocytes observed in L-TRF membranes according to group $(\mathrm{n}=10)$

Histologically, cell layers were completely separated in the L-TRF membranes, but sometimes small groups of a cell type were in other cell layers. Leukocytes were difficult to identify in some histological images because they were diffuse and interspersed with erythrocytes. Both fibrin and thrombocytes were easily identified. Fibrin was identified due to its appearance as a dense pink layer and thrombocytes were observed for their group arrangement. Figure 6 shows the histological aspects of Group 3 L-TRF membranes.

\section{Discussion}

Blood collection from the right jugular vein, which has greater calibre than the left one (Campbell, 1994), allowed quick transfer of the blood into a dry glass tube before clotting started. The success in PRF production is related to the speed of blood collection and transfer to the centrifuge; if that manipulation is not quick enough, diffuse polymerisation of the fibrin will occur and a small non-consistent clot will be formed (Dohan et al., 2006). 


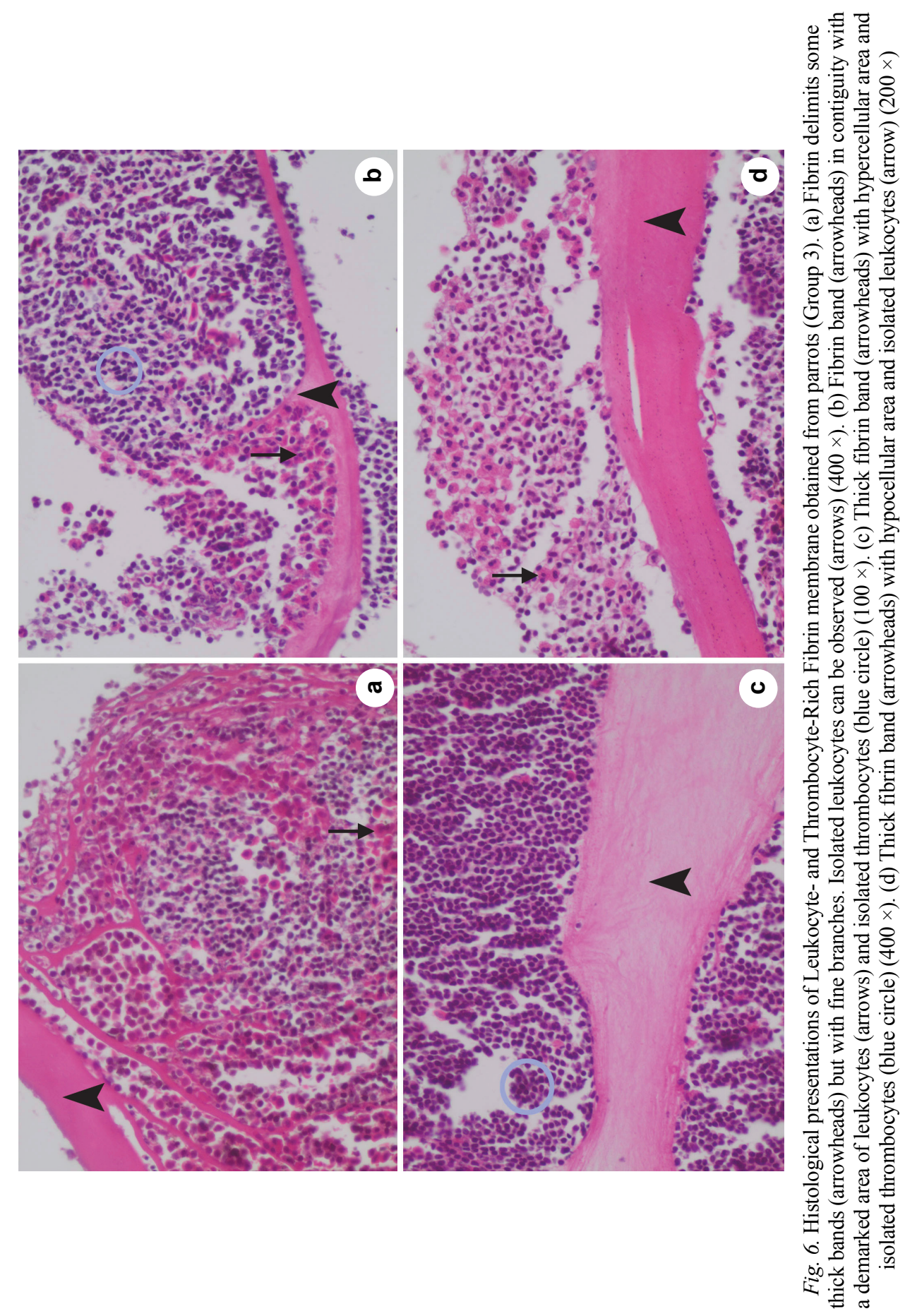


Centrifugation protocols and centrifuge characteristics influence the cells, fibrin architecture and growth factors in both L-PRF membranes and L-PRF clots (Ehrenfest et al., 2018). In the present study, the centrifugation was performed at $3000 \mathrm{rpm}$ for $10 \mathrm{~min}$ using a table-top centrifuge, which enabled the formation of a consistent blood clot.

Different centrifugation protocols have been reported for PRF preparation in humans, such as: the original PRF protocol or Choukroun's PRF, in which venous blood is centrifuged at $3000 \mathrm{rpm}$ for $10 \mathrm{~min}$ (Ehrenfest et al., 2010); Leukocyte- and Platelet-Rich Fibrin protocol, which uses $2700 \mathrm{rpm}$ for $12 \mathrm{~min}$ (Ehrenfest et al., 2018); Advanced PRF and low speed + time, which is performed for $8 \mathrm{~min}$ at $1300 \mathrm{rpm}$ (Fujioka-Kobayashi et al., 2017); and injectable formulation of PRF (i-PRF) produced in solution or gel at $700 \mathrm{rpm}$ for $3 \mathrm{~min}$ (Hartshorne and Gluckman, 2016b). Although centrifugation in this study simulates the original Choukroun protocol, leukocytes were observed in the histological analysis, suggesting a non-pure membrane, which was determined as L-TRF.

Manipulation was careful enough to preserve a small layer of $\mathrm{RBC}$ at the end of the clot, as indicated by other authors, as the portion of the blood clot attached to the fibrin clot contains stem cells (Hartshorne and Gluckman, 2016b). In addition, it has been reported that platelets are not evenly located on the surface and inside the PRF clot (Hartshorne and Gluckman, 2016b), as also observed in the histological analysis of the present study.

The PRF clot compressed between two layers of gauze becomes a membrane (Ehrenfest et al., 2009; Giannini et al., 2015), as observed in the present study. For human patients, specifically designed boxes (BoX grid) transform the clot into the membrane under sterile conditions (Hartshorne and Gluckman, 2016b); however, these have not yet been tested for birds. In all groups, the L-TRF membranes were flexible and apparently resistant. The three-dimensional fibrin matrix gives the PRF membrane several biomechanical characteristics including resistance against the pull of the suture (Giannini et al., 2015). However, biomechanical studies are necessary to determine the characteristics of avian L-TRF membranes.

In all groups, cell layers were completely separated in the L-TRF membranes on histological examination, but small groups of a cell type were occasionally observed in other cell layers. A study of fibrin network patterns of PRF clots in human patients reported the presence of an outermost layer of red blood cells, that segued into a dense fibrin layer containing platelets and red blood cells, and a lower layer constituted by loose fibrin with fewer platelets and red blood cells (Yajamanya et al., 2016).

Among active elements in human PRF associated with healing and tissue regeneration are fibrin, platelets, and cells such as leukocytes and stem cells (Hartshorne and Gluckman, 2016a). For this reason, the current study aimed to compare fibrin, leukocytes and thrombocytes between the membranes. As to the 
ratio of fibrin to cells, G4 showed the highest ratio whereas no differences were detected among the other groups. This finding is highlighted as fibrin serves as a support matrix and has direct influence on the biology of fibrin-based autologous biomaterials (Ehrenfest et al., 2018). In turn, the number of leukocytes/area did not differ among groups. Although leukocytes are responsible for antibacterial and osteoconductive activity (Hartshorne and Gluckman, 2016a), the importance of PRF biology remains controversial (Ehrenfest et al., 2009). In addition, despite the fact that in vitro studies have reported the superiority or inferiority of the preparations, they have not yet been validated in clinical trials (Hartshorne and Gluckman, 2016b).

The highest ratio of thrombocyte concentration/area occurred in G1, and the other groups showed similar numbers. In mammals, the number of platelets is an important data point due to the role platelet growth factors play in PRF biology (Ehrenfest, 2010; Hartshorne and Gluckman, 2016a). Extrapolation must be used with meticulous care, as the avian blood coagulation process is due to thrombocytes, which are nucleated cells instead of cytoplasmic fragments such as platelets (Campbell, 1994). Unlike mammalian platelets, avian thrombocytes originate from stem cells and not from megakaryocytes (Mitchell and Johns, 2008). The function of thrombocytes is still not fully understood, but together with haemostasis and thromboplastin production, they also present a phagocytic action (Mitchell and Johns, 2008; Jones, 2015). Thus, the highest number of thrombocytes in the macaw's membrane (Group 1) suggests a positive effect on PRF biology.

In conclusion, the centrifugation protocol allowed the production of LTRF membranes in the four bird species studied. Histologically, cell ratios were analogous in domestic chickens and parrots, while macaws had the highest ratio of thrombocytes. This study might serve as a basis for further clinical trials.

\section{Acknowledgements}

The authors are grateful to CNPq (National Council for Scientific and Technological Development - 301585/2017-2, Brasília, Distrito Federal, Brazil) and the National Institute of Science and Technology of Wildlife Science Center (WSC, Botucatu, São Paulo, Brazil).

\section{References}

Bielecki, T. and Ehrenfest, D. M. D. (2012): Platelet-Rich Plasma (PRP) and Platelet-Rich Fibrin (PRF): surgical adjuvants, preparations for in situ regenerative medicine and tools for tissue engineering. Curr. Pharm. Biotechnol. 13, 1121-1130.

Borie, E., Oliví, D. G., Orsi, I. A., Garlet, K., Weber, B., Beltrán, V. and Fuentes, R. (2015): Plateletrich fibrin application in dentistry: a literature review. Int. J. Clin. Exp. Med. 8, 7922-7929. 
Campbell, T. W. (1994): Hematology. In: Ritchie, B. W., Harrison, G. J. and Harrison, L. R. (eds) Avian Medicine: Principles and Applications. Wingers Publishing, Lake Worth. pp. 176-198.

Dhurat, R. and Sukesh, M. S. (2014): Principles and methods of preparation of platelet rich plasma: a review and author's perspective. J. Cutan. Aesthet. Surg. 7, 189-197.

Dohan, D. M., Choukroun, J., Diss, A., Dohan, S. L., Dohan, A. J. J., Mouhyi, J. and Gogly, B. (2006): Platelet-rich fibrin (PRF): A second-generation platelet concentrate. Part I: Technological concepts and evolution. Oral Surg. Oral Med. Oral Pathol. Oral Radiol. Endod. 101, e37-44.

Ehrenfest, D. M. D. (2010): How to optimize the preparation of leukocyte- and platelet-rich fibrin (L-PRF, Choukroun's technique) clots and membranes: introducing the PRF box. Oral Surg. Oral Med. Oral Pathol. Oral Radiol. Endod. 110, 275-278.

Ehrenfest, D. M. D., Del Corso, M., Diss, A., Mouhyi, J. and Charrier, J-B. (2010): Threedimensional architecture and cell composition of a Choukroun's platelet-rich fibrin clot and membrane. J. Periodontol. 4, 546-555.

Ehrenfest, D. M. D., Pinto, N. R., Pereda, A., Jiménez, P., Del Corso, M., Kang, B. S., Nally, M., Lanata, N., Wang, H-L. and Quirynen, M. (2018): The impact of the centrifuge characteristics and centrifugation protocols on the cells, growth factors, and fibrin architecture of a leukocyte- and platelet-rich fibrin (L-PRF) clot and membrane. Platelets 29, 171-184.

Ehrenfest, D. M. D., Rasmusson, L. and Albrektsson, T. (2009): Classification of platelet concentrates: from pure platelet-rich plasma (P-PRP) to leucocyte- and platelet-rich fibrin (L-PRF). Trends Biotechnol. 27, 158-167.

Forbes, N. A. and Guzman, D. S-M. (2017): Avian Medicine and Surgery: Self-assessment Color Review. CRC Press, Boca Raton.

Fujioka-Kobayashi, M., Miron, R. J., Hernandez, M., Kandalam, U., Zhang, Y. and Choukroun, J. (2017): Optimized platelet-rich fibrin with the low-speed concept: growth factor release, biocompatibility, and cellular response. J. Periodontol. 88, 112-121.

Giannini, S., Cielo, A., Bonanome, L., Rastelli, C., Derla, C., Corpaci, F. and Falisi, G. (2015): Comparison between PRP, PRGF and PRF: lights and shadows in three similar but different protocols. Eur. Rev. Med. Pharmacol. Sci. 19, 927-930.

Hartshorne, J. and Gluckman, H. (2016a): A comprehensive clinical review of Platelet Rich Fibrin (PRF) and its role in promoting tissue healing and regeneration in dentistry. Part 1: Definition, development, biological characteristics and function. Int. Dentistry Afr. Ed. 6, 14-24.

Hartshorne, J. and Gluckman, H. (2016b): A comprehensive clinical review of Platelet Rich Fibrin (PRF) and its role in promoting tissue healing and regeneration in dentistry. Part II: Preparation, optimization, handling and application, benefits and limitations of PRF. Int. Dentistry Afr. Ed. 6, 34-48.

Hartshorne, J. and Gluckman, H. (2016c): A comprehensive clinical review of Platelet Rich Fibrin (PRF) and its role in promoting tissue healing and regeneration in dentistry. Part III: Clinical indications of PRF in implant dentistry, periodontology, oral surgery and regenerative endodontics. Int. Dentistry Afr. Ed. 6, 64-79.

Jones, M. P. (2015): Avian hematology. Clin. Lab. Med. 35, 649-659.

Lansdown, D. A. and Fortier, L. A. (2017): Platelet-Rich Plasma: formulations, preparations, constituents, and their effects. Oper. Tech. Sports Med. 25, 7-12.

Mautner, K., Malanga, G. A., Smith, J., Shiple, B., Ibrahim, V., Sampson, S. and Bowen, J. E. (2015): A call for a standard classification system for future biologic research: the rationale for new PRP nomenclature. PM \& R 7, s53-s59.

Miron, R. J., Zucchelli, G., Pikos, M. A., Salama, M., Lee, S., Guillemette, V., Fujioka-Kobayashi, M., Bishara, M., Zhang, Y., Wang, H. L., Chandad, F., Nacopoulos, C., Simonpieri, A., Aalam, A. A., Felice, P., Sammartino, G., Ghanaati, S., Hernandez, M. A. and Choukroun, J. (2017): Use of platelet-rich fibrin in regenerative dentistry: a systematic review. Clin. Oral Investig. 21, 1913-1927. 
Mitchell, E. B. and Johns, J. (2008): Avian hematology and related disorders. Vet. Clin. North Am. Exot. Anim. Pract. 11, 501-522.

Mudroňová, D., Kožár, M., Molnár, L. and Trbolová, A. (2014): Viability and discrimination of avian peripheral blood mononuclear cells and thrombocytes intended for improvement of wound healing in birds. Acta Vet. Hung. 62, 334-339.

Saluja, H., Dehane, V. and Mahindra, U. (2011): Platelet-rich fibrin: A second generation platelet concentrate and a new friend of oral and maxillofacial surgeons. Ann. Maxillofac. Surg. 1, $53-57$.

Sant'Ana, P. G., Batah, S. S., Leão, P. S., Teodoro, W. R., Souza, S. L. B., Ferreira Mota, G. A., Vileigas, D. F., Silva, V. L., Campos, D. H. S., Okoshi, K., Capelozzi, V. L., Cicogna, A. C. and Fabro, A. T. (2018): Heart remodeling produced by aortic stenosis promotes cardiomyocyte apoptosis mediated by collagen V imbalance. Pathophysiol. 25, 373-379.

Yajamanya, S. R., Chatterjee, A., Babu, C. N. and Karunanithi, D. (2016): Fibrin network pattern changes of platelet-rich fibrin in young versus old age group of individuals: A cell block cytology study. J. Indian Soc. Periodontol. 20, 151-156. 